\title{
Multi-stage Multi-fidelity Information Correction for Artificial Neural Network Based Meta-modelling
}

\author{
$1^{\text {st }}$ Ben Parsonage \\ Dept. of Mechanical and Aerospace Engineering \\ University of Strathclyde \\ Glasgow, United Kingdom \\ ben.parsonage@strath.ac.uk
}

\author{
$2^{\text {nd }}$ Christie Alisa Maddock \\ Dept. Mechanical and Aerospace Engineering \\ University of Strathclyde \\ Glasgow, United Kingdom \\ christie.maddock@strath.ac.uk
}

\begin{abstract}
Multi-fidelity meta-modelling has become a popular means of efficiently distributing computational resource across various levels of simulation fidelity to obtain numerically accurate predictions of an expensive function. Such techniques have significant potential within an engineering design paradigm incorporating either many-query analyses or outer-loop applications.

This paper presents a hybrid parametric/non-parametric information correction method incorporating the sequential application of several distinct stages within an artificial neural network based surrogate framework. The proposed methodology may be used to correct any domain encompassing set of low-fidelity input/output correspondence using a small subset of high-fidelity samples. A global surrogate can then be generated via a doubleloop ANN hyper-parameter selection and training procedure.

To demonstrate the effectiveness of the proposed metamodelling approach, the aerodynamic response prediction of a parametrized waverider-based re-entry vehicle is examined. Results suggest that the incorporation of multiple corrective stages leveraging low-fidelity data can offer significant improvements in computational efficiency when modelling the expensive highfidelity function compared with single stage correction. The costs to achieve global accuracy are examined and compared across single/multi-stage variants, with consideration given to surrogate construction and evaluation. Results are compared both with the low-fidelity approximation and a surrogate of the 'true' response built using only the high-fidelity samples available to the corrective method.
\end{abstract}

Index Terms - multi-fidelity, surrogate, correction, ANN

\section{INTRODUCTION}

The advancement of computational processing capability has significant implications for the design of complex multidisciplinary systems [1]. The ability to simulate physical processes to an increasingly high degree of fidelity, coupled with an upwards trend in system performance requirements, has promoted a widespread desire to include high-fidelity (HF) subsystem analyses at earlier stages in the design process [2], [3]. Such practice, in departure from the traditional methodology of upgrading modelling fidelity at a rate proportional to high-level developmental progress [4], [3], has the potential to identify complex subsystem interactions at a sufficiently early stage as to mitigate the economic implications of late stage alterations and ultimately achieve previously unattainable levels of performance [3].
Cause for concern is the high dependence on reliable simulation of individual subsystems. Indeed, the consequences of completing a formal multidisciplinary design study with inaccurate or misleading models may eradicate any developmental/financial savings gained by including such analyses in the first place [5]. Unfortunately, the benefits of advancing computational power (commonly cited in relation to Moore's law, see [6]) are inexorably tempered by increasing simulation complexity [7], [1]. Many advanced numerical methods, such as Computational Fluid Dynamics (CFD) and Finite Element Analysis (FEA), still typically require significant computational resource, even for isolated analyses. Such costs limit their use within numerical optimisation studies, for which subsystem models may be evaluated many thousands of times.

A traditional strategy to mitigate this incompatibility is to approximate the expensive simulation with a cheap to evaluate surrogate model (or meta-model). This simple conjecture has prompted a wide range of developmental areas across science and engineering [5], [8], [9]. Indeed, Simpson argues (in defence of Moore's law) that the decreasing cost ratio of surrogate generation relative to the cost of the required HF analyses has promoted the development and application of increasingly sophisticated meta-modelling techniques [1].

Directly constructing such surrogates with HF sampling can nonetheless remain prohibitively expensive [10], especially when underlying non-linearities or multi-modality necessitate a higher number of samples for a useful approximation. A method that has received considerable attention in recent years is the use of multi-fidelity (or variable fidelity) surrogates. That is, models leveraging the response trends of one or more inexpensive, lower quality models in conjunction with a small number of HF evaluations to accurately mimic the expensive function response at a fraction of the cost.

This paper presents an automated multi-fidelity (MF) model generation method intended for use within many-query/outerloop applications. The method utilises the sequential application of three individual response correction techniques: parametric scaling, relative response mapping and additive discrepancy modelling within an artificial-neural-network (ANN) surrogate based framework. The method is found to outperform selected single and 2-stage variants in terms of attainable prediction accuracy relative to the number of HF samples. 
The remainder of this paper is organised as follows. Section II briefly reviews MF model management and ANN applications. Section III introduces the mathematical basis behind the ANN fitting technique employed in this paper. Section IV presents the proposed MF meta-modelling approach, including overall concept and mathematical formulation. In sections $\mathrm{V}$ and VI, this approach is applied to the aerodynamic prediction of a parametrized waverider-based re-entry vehicle. Section VII presents the associated cost savings, and finally section VIII presents the author's conclusions and recommendations.

\section{BACKGROUND}

\section{A. Multi-fidelity model management}

MF modelling assumes that for an accurate yet computationally expensive model there exists at least one alternative model that for a significantly reduced cost can represent features of interest and/or general trends of the underlying physical process [11]. Two key applications can be described via the distinction between many-query analyses, in which global accuracy is promoted across constituent subsystem models, and outer-loop applications, in which local accuracy is promoted within the vicinity of the desired/expected solution [11]. A MF process is conducted via two distinct stages:

1) The selection/generation of appropriate low-fidelity (LF) models (or surrogates thereof).

2) The implementation of an appropriate model management strategy that distributes computational power amongst information sources to establish numerical accuracy or guide an outer-loop towards convergence.

Given the inherent reliance of correction quality on the correlation between the LF and HF approximations [12], it becomes important to examine the LF modelling approaches available for a given physical process. LF models may be derived according to three high-level classifications [11]; simplification, projection and data-fit. Simplification refers to models constructed via simplifying assumptions relative to a HF counterpart. For example, simplifying the analysis model (e.g. coarse instead of refined mesh discretization [13], neglected non-linearities or an early stopping criteria [14]), simplifying the domain/level of abstraction (e.g. 2D versus 3D [4], additional boundary conditions [15] or simplified geometry [16]) or simplifying the mathematical/physical description (e.g. Euler non-cohesive instead of viscous Navier-Stokes [17]). Projection based models take advantage of problem structure, as opposed to expert domain knowledge [11] to 'project' the input/output relationship of the HF model onto a lower-dimensional subspace, thus exploiting general trends and features. Methods include Proper Orthagonal Decomposition (POD), Reduced Basis Modelling, Centroidal Voronoi Tessellation (CVT) and Krylov Subspace methods [8], [11]. Finally, data-fit models are derived directly from a finite set of input/output correspondence. An advantage here is that there is no need for any additional formulation, samples may be collected directly using the existing simulation/model. Naturally then, traditional interpolation or meta-modelling techniques such as Polynomial Response Surface Modelling (RSM), Radial Basis Functions, Kriging, Support Vector Regression and Artificial Neural Networks may be employed [18], [9] (assuming sufficient samples are available).

MF methods differ in their approach to combine distinct levels of fidelity. Adaptive methods directly correct/enhance an existing LF model (or set of models) using selective HF samples [19], fusion methods combine separate levels of fidelity into a single surrogate response, without any explicit correction [20], and filtering methods employ a hierarchical management of separate models, typically dependant on acceptable relative accuracy or related criteria [21]. Each of the preceding techniques may be applied to an optimisation problem via an appropriate model management framework. Common approaches include utilising one or more LF/MF models to accelerate the search for the global optimum [22], leveraging an adaptively corrected MF model within a trust region model management scheme [23], or directly optimising an MF approximation of the objective function [10]. Recent progress includes the work of Han et al [24] to extend the Hierarchical Kriging methodology to a 'multi-level' case incorporating an arbitrary number of model fidelities, the hybrid additive/multiplicative bridge function applied by Fischer et al [2] for which a weighted combination of two discrepancy functions is determined via a Bayesian update technique implemented within a trust-region model management optimisation framework, and the work of Zhou et al [25], in which their two-stage adaptive MF surrogate generation method incorporating a linear parameter extraction is integrated into a Multi-Objective Genetic Algorithm (MOGA).

Various software implementations of established MF surrogate modelling methods are available, such as the MATLAB SUrrogate MOdelling (SUMO) toolbox [26] and Automated Learning of Algebraic Models for Optimisation (ALAMO) [27]. Less prevalent are packages tailored to MF conceptual aircraft design. A notable example is the Stanford University Aerospace Vehicle Environment (SUAVE) [28].

\section{B. Artificial Neural Networks}

An Artificial Neural Network (ANN) is a collection of simple computational units (neurons) arranged into an interconnecting parallel processing structure [29]. A network may be 'trained' according to a finite set of corresponding input/target vectors. A suitably trained ANN may perform tasks such as pattern recognition, identification, classification, system control and function approximation (non-linear regression)[29]. In aerospace, ANNs have been used for the estimation of aerodynamic coefficients [30], space vehicle design and trajectory optimisation [31], [32], turbo-machinery blade optimisation [33], wing design [34], flow control, aeroelasticity, and interpolation of wind tunnel data [18].

Despite wide applicability, a recent study found that ANNs account for only $4 \%$ of peer-reviewed MF modelling studies[8]. Bakr et al [35] present a space-mapping based neuro-modelling optimisation technique in which the HF optimum is found by replacing the LF model at each iteration with 
an adaptively-sized ANN trained using the HF samples from the previous iteration. Leary [36] introduced the 'knowledgebased-neural-network' (KBNN) technique as a means of incorporating LF data into the training procedure of a limited set of HF samples via scaling/translation based parameter mapping. Minisci and Vasile [32] estimate aerodynamic coefficients using a MF ANN first trained on a global set of samples given by a simplified analytical model, then iteratively refined with CFD data scheduled via evolution control. LF samples are sequentially discarded should they fall outwith a predefined applicability region relative to new HF points.

\section{DOUBLE-LOOP TRAINING}

This paper employs a generic training methodology for multi-layer feed-forward networks, largely adapted from the recommendations of Heath [37]. The method is an attempt to automatically determine the optimal parameters of a standard MLP architecture implied by the Kolmogorov theorem [38]. The method utilises both Bayesian Regularization and an early-stopping criteria implemented using the MATLAB ${ }^{\circledR}$ Neural Network Toolbox [29]. The number of samples allocated to the training, validation and testing sets, $n_{t r n}, n_{v a l}$ and $n_{t s t}$, are determined via the default data division ratios:

$$
\left[\begin{array}{l}
n_{t r n} \\
n_{v a l} \\
n_{t s t}
\end{array}\right]=n_{s}\left[\begin{array}{c}
\phi_{t r n} \\
\phi_{v a l} \\
\phi_{t s t}
\end{array}\right]=n_{s}\left[\begin{array}{c}
0.7 \\
0.15 \\
0.15
\end{array}\right]
$$

where the number of samples $n_{s}$ for an input vector of dimension $d_{i n}$ is chosen such that:

$$
10 d_{i n} \leq n_{t r n} \leq 30 d_{i n}
$$

The initialization process allocates random initial weights and biases to the network and divides the available input data into training, validation and testing sets according to (1). Random initialization coupled with a gradient-based backpropagation training algorithm cannot guarantee convergence to the optimal parameters [29], introducing a degree of variability in the network solution. While some authors advocate re-training the network multiple times, or averaging the response from multiple networks, these methods still ultimately rely on a certain degree of user discretion. The number of unknown parameters (weights and biases) $N_{w}$ is given by:

$$
N_{w}=H\left(d_{\text {in }}+1\right)+d_{\text {out }}(H+1)
$$

where $H$ is the number of neurons in the hidden layer. The number of training equations required for a given training set is given by:

$$
N_{\text {trneq }}=n_{\text {trn }} * d_{\text {out }}
$$

where the number of samples in the training set $n_{t r n}$ is consistent with (1). To avoid over-fitting,

$$
N_{w} \leq N_{\text {trneq }}
$$

From equations (3) and (4) it is clear that (5) will always be true provided the number of neurons $H$ is less than or equal to an upper bound $H_{u b}$ :

$$
H_{u b}=\left\lceil\frac{d_{\text {out }}\left(n_{\text {trn }}-1\right)}{d_{\text {in }}+d_{\text {out }}+1}\right\rceil
$$

To reduce the risk of poor generalisation, it is of interest to minimise the number of hidden layer neurons. A doubleloop strategy for network training and parameter selection is therefore employed. In the outer loop, the number of neurons $H$ is varied in 10 near-equally spaced divisions between a lower bound of 0 (representing a linear response) and the upper bound $H_{u b}$. The inner loop performs 10 random initializations of the network weights, biases and data divisions, before sequentially training each network to convergence. The objective is to determine the network parameters that yield an acceptable ratio of mean-square-error to mean-target-variance for random subsets of non-training data [37]. A normalized performance criteria relating predictions $\boldsymbol{\alpha}$ to targets $\mathbf{t}$ is utilised:

$$
R^{2}=1-\frac{\frac{1}{N} \sum_{i=1}^{N}\left(t_{i}-\alpha_{i}\right)^{2}}{\frac{1}{N} \sum_{i=1}^{N}\left(t_{i}-\bar{t}\right)^{2}}
$$

A minimum performance criteria is defined as the successful modelling of $99 \%$ of the target subset variance $\left(R^{2} \geq 0.99\right)$. Therefore, the network that achieves this criteria for a minimum number of hidden layer neurons is selected.

\section{PROPOSED APPROACH}

\section{A. Motivation}

This section presents the decomposition of a direct information correction process into three fundamental stages, each leveraging an alternate aspect of a correlated LF response. Each stage may be applied individually or sequentially to correct a domain encompassing set of LF responses with a small number of HF samples. A globally approximating surrogate suitable for many-query/outer-loop applications can then be constructed via the automated hyper-parameter selection and training procedure detailed in section III.

The aim is to provide a global representation of the HF response meeting the requirements of zero- and first-order consistency [23] to an acceptable degree with a significantly reduced computational cost compared with constructing a surrogate of similar accuracy using exclusive HF sampling.

\section{B. Formulation}

A generic model is defined as a function $\mathbf{f}: \mathbf{X} \rightarrow \mathbf{Y}$ that for a given input $\mathbf{x} \in \mathbf{X}$ produces an output $\mathbf{y} \in \mathbf{Y}$ where $\mathbf{X} \subseteq \mathbb{R}^{d_{\text {in }}}$ is the input domain with dimension $d_{\text {in }} \subset \mathbb{N}$ and $\mathbf{Y} \subset \mathbb{R}^{d_{\text {out }}}$ is the output domain with dimension $d_{\text {out }} \subset \mathbb{N}$ :

$$
\mathbf{x}^{(i)} \mapsto \mathbf{y}^{(i)}=\mathbf{f}\left(\mathbf{x}^{(i)}\right), \quad i=1,2, \ldots, n_{s}
$$

In this context, $\mathbf{f}(\mathbf{x})$ may represent the direct evaluation of a subsystem model or the evaluation of an appropriate surrogate approximation. Thus HF and LF models are notated simply as $\mathbf{f}_{H}: \mathbf{X} \rightarrow \mathbf{Y}$ and $\mathbf{f}_{L}: \mathbf{X} \rightarrow \mathbf{Y}$, and a generic surrogate model $(\mathrm{SM})$ as $\mathbf{f}_{S}: \mathbf{X} \rightarrow \mathbf{Y}$. Let $\mathbf{x}^{(i)}, i=1, \ldots, M$ denote the set of $M$ design points. Directly collocated LF/HF samples are assumed, although such a correspondence may be obtained via surrogate estimation [39]. Therefore the set of $N$ HF samples is defined as $\mathbf{x}_{H}^{(i)}, i=1, \ldots, N$ where $\mathbf{x}_{H} \in \mathbf{x}$. 
For ease of notation, the following discussion is limited to the case of correcting an unspecified LF function $\mathbf{f}_{L}$ via selective samples of a HF counterpart $\mathbf{f}_{H}$. In reality, the sequential application of stages necessitates that the LF function for each subsequent stage is of course the MF output from the previous stage.

\section{Parameter extraction}

An approximate relationship between an LF and HF response (of dimension $d_{\text {out }}$ ) is defined as a quantifiable set of scaling parameters of the linear form [9]:

$$
\mathbf{f}_{S}(\mathbf{x}, \mathbf{p})=\mathbf{a f}_{L}(\mathbf{x})+\mathbf{b}
$$

where $\mathbf{a}$ and $\mathbf{b}$ are vectors of $d_{\text {out }}$ unbounded scaling coefficients, and $\mathbf{p}(\mathbf{a}, \mathbf{b})$ is found by formulating (9) into a parameter extraction problem:

$$
\mathbf{p}=\arg \min _{\mathbf{q}} \frac{1}{\sigma} \sqrt{\frac{1}{N} \sum_{k=1}^{N}\left[\mathbf{f}_{S}\left(\mathbf{x}_{H}^{(k)}, \mathbf{q}\right)-\mathbf{f}_{H}\left(\mathbf{x}_{H}^{(k)}\right)\right]^{2}}
$$

where $\sigma$ is the standard deviation of the HF samples.

\section{Relative response mapping}

An alternate approach is to quantify the change in LF response between each pair-wise combination of $M$ design points $\mathbf{x}$ and $N \mathrm{HF}$ samples $\mathbf{x}_{H}$. The resulting translation matrix $\mathbf{t}$ is notated as:

$$
\mathbf{t}_{i, j}=\mathbf{f}_{L}\left(\mathbf{x}^{(i)}\right)-\mathbf{f}_{L}\left(\mathbf{x}_{H}^{(j)}\right) \quad\left\{\begin{array}{c}
i=1, \ldots, M \\
j=1, \ldots, N
\end{array}\right.
$$

The HF response at each design point $\mathbf{x}^{(i)}$ is predicted via:

$$
\mathbf{f}_{S}\left(\mathbf{x}^{(i)}\right)=\frac{\sum\left[\left(\mathbf{f}_{H}\left(\mathbf{x}_{H}^{(j)}\right)+\mathbf{t}_{i, j}\right) * \mathbf{w}_{i, j}\right]_{j=1, \ldots, N}}{\sum\left[\mathbf{w}_{i, j}\right]_{j=1, \ldots, N}}
$$

The weight factor $\mathbf{w}_{i, j}$ is defined by the euclidean norm between each design point and each HF sample:

$$
\mathbf{w}_{i, j}=1-\sqrt{\sum_{k=1}^{d_{i n}}\left[\hat{\mathbf{x}}^{(i, k)}-\hat{\mathbf{x}}_{H}^{(j, k)}\right]^{2}}
$$

where $d_{i n}$ is the dimension of the input and $\hat{\mathbf{x}}$ denotes the scaled form of input $\mathbf{x}$ conforming to $\hat{\mathbf{x}}^{(i, k)} \in[0,1]$ for all $k=1, \ldots, d_{i n}$. The weight factor is constrained to $\mathbf{w}_{i, j} \in$ $[0,1]$ to negate the influence of a design point lying far from a particular HF sample point.

\section{E. Neural Network bridge function}

The final stage constructs an ANN surrogate of the additive discrepancy between responses, defined as a matrix of translation vectors $\mathbf{t}$ :

$$
\mathbf{t}_{j}=\mathbf{f}_{L}\left(\mathbf{x}_{H}^{(j)}\right)-\mathbf{f}_{H}\left(\mathbf{x}_{H}^{(j)}\right), \quad j=1, \ldots, N
$$

To mitigate the implications of an insufficient input set of $N$ samples on training performance, an augmented dataset of translation vectors $\tilde{\mathbf{t}}$ may be defined as:

$$
\tilde{\mathbf{t}}=[\mathbf{t}, \mathbf{a}], \quad \mathbf{a}_{j}=\mathbf{b}_{j}+w \boldsymbol{\theta}_{j}, \quad j=1, \ldots, n_{a u g}
$$

where $\mathbf{b} \subset \mathbf{t}, w$ is a scalar weighting factor (default $1 \%$ ), $\boldsymbol{\theta}$ is a vector of random values $\boldsymbol{\theta} \in[-1,1]$ and the number of augments $n_{\text {aug }}=n_{\text {desired }}-N$. The minimum input set size $n_{\text {desired }}=30 d_{i n} / \phi_{t r n}$, where $\phi_{t r n}$ is consistent with (1). The resulting set of translation vectors is mapped to a continuous function via the double-loop training procedure detailed in Section III. Performance is additionally examined by evaluating the prediction accuracy obtained by applying the translation network across the original LF response data. The network exhibiting the strongest correction quality and training accuracy is therefore selected.

\section{TEST}

This section considers the aerodynamic response of a waverider-based re-entry vehicle. The proposed MF metamodelling approach is used to generate approximations of lift and drag coefficients, $C_{L}$ and $C_{D}$, as functions of several independent design variables describing vehicle shape and operational conditions at two distinct levels of fidelity.

\section{A. Geometry parametrization}

A waverider may be parametrized according to the 2D power law equations [40] shown in Fig. 1. The curvatures of the planform $p$ and the upper surface $u$ are given by:

$$
\begin{gathered}
y_{p}=A x^{n} \\
y_{u}=B\left(z_{u}\right)^{n} \rightarrow z_{u}=\left(\frac{y_{u}}{B}\right)^{\frac{1}{n}}
\end{gathered}
$$

where the exponent $n \in[0,1]$ and the positive scaling coefficients $A$ and $B$ are given by:

$$
\begin{gathered}
A=w / 2 l^{n} \\
B=a / \tan ^{n}(\beta)
\end{gathered}
$$

The curvature of the lower surface with an attached shock is given by:

$$
z_{l}=x \tan \theta+(y / A)^{1 / n}(\tan \beta-\tan \theta)
$$

Vehicle height $h$ and reference surface area $S_{\text {ref }}$ are given by:

$$
\begin{gathered}
h=l \tan \beta \\
S_{\text {ref }}=w l /(n+1)
\end{gathered}
$$

The geometric design vector $\mathbf{d}(l, w, n, \theta)$ is defined via the variable bounds given in table I [32]. By parametrising in this manner, small variations in $\mathbf{d}$ can correspond to significant change in the resulting geometry [40]. Three representative examples, the maximum, mean and minimum values of $\mathbf{d}$, are shown in Fig. 1.

TABLE I: Waverider parameters

\begin{tabular}{|l|l|}
\hline Parameter & Bounds \\
\hline Length, $l[\mathrm{~m}]$ & {$[2.9,4.2]$} \\
Width, $w[\mathrm{~m}]$ & {$[1,2]$} \\
Exponent, $n$ & {$[0.2,0.7]$} \\
Wedge angle, $\theta[\mathrm{deg}]$ & {$[7,11]$} \\
Shock angle, $\beta$ [deg] & 12 \\
\hline
\end{tabular}




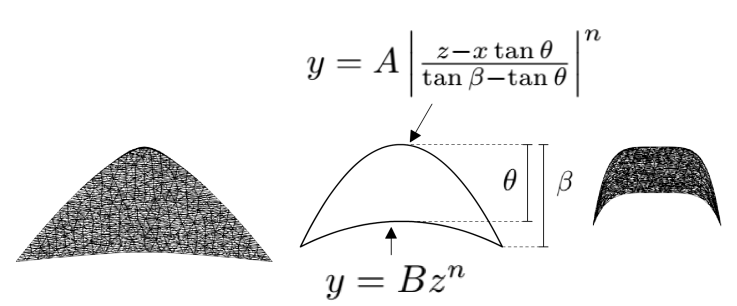

(a) $\mathrm{x}$-axis

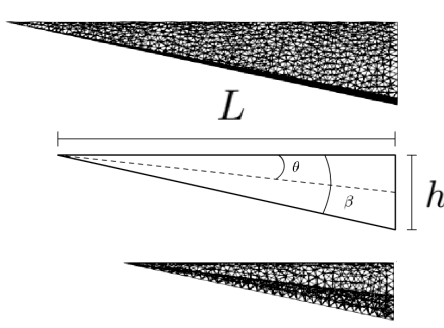

(b) y-axis

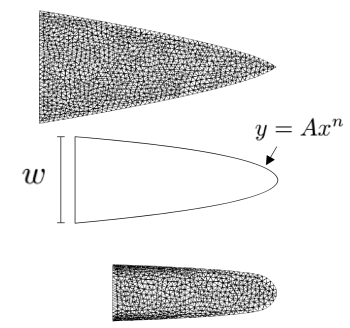

(c) z-axis

Fig. 1: Waverider geometry parameterization

1) GMSH: The open source meshing tool GMSH (v4.6.0) [41] is selected for geometry generation. Equations (16), (17) and (20) are used to define 10 equally spaced cross sections along the longitudinal axis of the vehicle according to the user input vector d. Cross sections are combined into a coherent volume using the ThruSections command included within the OpenCascade kernel. A solid surface mesh may then be exported as a stereolitographic (.stl) file.

To produce a volume mesh suitable for CFD analyses, a half-cylindrical far-field of length $l c=20 \mathrm{~m}$, radius $r=10 \mathrm{~m}$ and spherical input/output caps each of radius $r=10 \mathrm{~m}$ is created around the surface mesh, with the y-symmetry plane bisecting the vehicle geometry along the longitudinal axis, see Fig. 2. A single volume mesh containing the vehicle half surface is created via Boolean subtraction. A simplified boundary mesh definition is incorporated by specifying a rectangular vehicle-centered bounding box with an internal mesh element size of $0.075 \mathrm{~m}$, external mesh element size of $2 \mathrm{~m}$ and a transition gradient layer of thickness $t=10 \mathrm{~m}$. The vehicle surface mesh is comprised of between 2,000 and 3,200 vertices and between 3,500 and 6,000 triangular elements. Likewise, the CFD volume mesh of around 275,000 elements and 46,000 nodes. Input, output, far-field and vehicle surface are labelled accordingly and the volume mesh is exported in the native SU2 format.

\section{B. Low-fidelity aerodynamics}

LF aerodynamics are computed using the Free Open Source Tool for Re-Entry of Asteroids and Debris (FOSTRAD) developed by the University of Strathclyde [42]. FOSTRAD is an LSI based aerodynamic/aerothermodynamic computation tool for arbitrary geometries in hypersonic continuum or freemolecular flow, intended for use within trajectory propagators and uncertainty analyses tools for re-entry scenarios [43], [44].

FOSTRAD considers solid surface meshes of triangular elements (panels). Modified Newtonian Theory and the Schaaf and Chambre analytic model are used to approximate continuum and free-molecular aerodynamic coefficients respectively [42]. Transitional regime aerodynamics, defined in [42] as $10^{-4}<K n<100$, are approximated using a sigmoid (base 10) bridging function. FOSTRAD contains a vectorized occlusion culling algorithm for panel shadowing determination [44], used to detect and remove panels hidden relative to the oncoming flow.

\section{High-fidelity aerodynamics}

HF simulations are conducted using the Stanford University Unstructured Code (SU2) [45]. The 3-dimensional RANS equations are employed with a standard SST turbulence model and a FVM discretization. The JST scheme is used in conjunction with a second-order scalar upwind discretization and Venkatakrishnan's limiter to model convective fluxes. The gradients of the spatial flow variables, required to evaluate viscous fluxes, are calculated using the Green-Gauss method with a CFL number of 1 . The linear system is solved using the GMRES method with an error tolerance of $1 e^{-10}$ and the steady state solution is achieved using the Euler implicit method for time integration. A Cauchy convergence criteria is applied to the Drag function after iteration 10. Convergence is defined as the reduction of the residuals by five orders of magnitude with respect to their initial values, within a maximum number of iterations fixed at 5,000.

\section{Method}

The coefficients of lift and drag, $C_{L}$ and $C_{D}$, for the vehicle defined by design vector $\mathbf{d}(l, w, n, \theta)$ are predicted for an operational range given by Mach number $2 \leq M a \leq 30$, angle of attack $0^{\circ} \leq \alpha \leq 20^{\circ}$ and altitude $10^{3} \mathrm{~m} \leq h \leq 10^{5} \mathrm{~m}$. An independent HF sample set is gradually increased from 2 to 20 samples, approximately corresponding to MF response convergence. The LF sample set is fixed at $M=30 d_{i n}$ given the general assumption that the cost of the LF model is negligible in comparison to that of the HF model. All samples are selected via LHS.

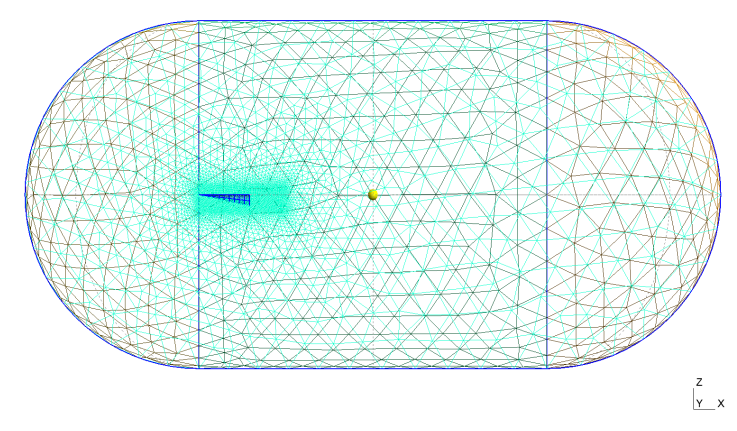

Fig. 2: CFD far-field $l=40 m, r=10 m$ 


\section{RESULTS}

To demonstrate cross-domain applicability, eight boundary configurations of $\mathbf{d}(l, w, n, \theta)$ are examined in addition to the complete domain $\mathbf{x}(M a, \alpha, h, \mathbf{d})$. Corrective stages are organised into sequential combinations according to applicability constraints relating to the type of information each may utilise. Accuracy metrics are determined via an independent set of HF responses. Results are averaged over 100 separate analyses.

Tables II and III show the average values of $N$ required to achieve MFSM accuracies of $R^{2}>90 \%$ and $R^{2}>95 \%$ for each geometric case (normalised to $[0,1]$ ). The parametric (P) stage consistently achieves $R^{2}>90 \%$ for $C_{D}$ in relatively few samples, suggesting a high correlation amenable to simple linear scaling/positioning. While the addition of successive stages merely preserves rather than improves this performance, the benefits are clear when considering the P-stage prediction of $C_{L}$, by contrast initially quite poor. For example, the relative (R) stage is mostly unable to produce an effective correction given it considers only the shaping of the response rather than it's direct correction. Yet, preconditioning with the P-stage (thus PR) largely eliminates the observed instability in $C_{L}$ while preserving $C_{D}$. This can be seen to a further extent with the parametric/additive (PA) method. In this case, not only is $C_{D}$ preserved, but $C_{L}$ reaches comparable levels, thus stronger overall than either stage individually. In fact, table II shows PA and PRA to be very similar, achieving identical results for $15 / 18$ cases, PRA improving only the remaining 3 . Greater variation can be seen in table III, in which PRA offers an improvement over PA in 9/18 cases, identical performance in 8 and only a marginal decrease in the remaining case.

This can be accounted for by considering the A-stage preconditioning. In table II, the P-stage achieves global minimum values for 9/18 cases. PR retains these minima and improves the remaining 9 cases, 3 of which become global minima themselves. In table III, the P-stage achieves global minima in only 4/18 cases. Again, PR retains these minima and similarly improves a further 10 cases, 3 reaching global minimum values. This marginal increase in improvement could be due to the greater resolution of the R-stage correction given a larger $N$. The A-stage is the only stage that aims to fully eliminate sample point discrepancy in a non-destructive manner (i.e. zero correction at points of zero discrepancy), thus particularly

TABLE II: $N$ required for $R^{2}>90 \%\left[C_{L}, C_{D}\right]$

\begin{tabular}{|c|c|c|c|c|c|c|c|c|}
\hline d & $\mathbf{P}$ & $\mathbf{R}$ & $\mathbf{A}$ & PR & RA & PA & $\overline{\text { PRA }}$ & $\overline{\text { HFSM }}$ \\
\hline $\begin{array}{lllll} & 0 & 0 & 0\end{array}$ & 6,3 & 4,4 & 5,7 & 4,3 & 4,5 & $\mathbf{3 , 3}$ & 3,3 & 5,7 \\
\hline $\begin{array}{llllll}0 & 0 & 1 & 1\end{array}$ & 4,2 &,-- & 4,7 & 4,2 & $\mathbf{4 , 8}$ & 4,2 & 4,2 & 5,6 \\
\hline $\begin{array}{lllll}0 & 1 & 1 & 0\end{array}$ &,- 4 & $18,-$ & 9,9 & 10,4 & 6,9 & $\mathbf{5 , 5}$ & 5,4 & 8,7 \\
\hline $\begin{array}{lllll}0 & 1 & 0 & 1\end{array}$ & 19,3 &,-- & 5,8 & 4,3 & 5,8 & 4,3 & 4,3 & 5,6 \\
\hline $\begin{array}{llll}10 & 10\end{array}$ & 5,3 & $5,-$ & 4,8 & 4,3 & 4,7 & 3,3 & 3,3 & 5,6 \\
\hline $\begin{array}{llll}1 & 0 & 0 & 1\end{array}$ & 4,3 & $11,-$ & 4,7 & 3,3 & 4,8 & 3,3 & 3,3 & 5,6 \\
\hline $\begin{array}{llll}1100 & 0\end{array}$ &,- 5 & 9,14 & 8,10 & 8,4 & 5,4 & 5,5 & $\mathbf{5 , 5}$ & 7,8 \\
\hline $\begin{array}{llll}1 & 1 & 1 & 1\end{array}$ &,- 3 &,-- & 6,7 & 5,3 & 5,9 & 5,3 & 4,3 & 6,6 \\
\hline $\mathbf{x}$ &,- 3 &,-- & 16,- & 9,3 & 10,- & 9,3 & 6,3 & 10,14 \\
\hline
\end{tabular}

TABLE III: $N$ required for $R^{2}>95 \%\left[C_{L}, C_{D}\right]$

\begin{tabular}{|c|c|c|c|c|c|c|c|c|}
\hline d & $\mathbf{P}$ & $\mathbf{R}$ & $\mathbf{A}$ & PR & RA & PA & PRA & HFSM \\
\hline $\begin{array}{lllll}0 & 0 & 0 & 0\end{array}$ &,- 10 &,,-- & 6,13 & 8,5 & 6,11 & 6,6 & 5,5 & 6,9 \\
\hline $\begin{array}{llllll}0 & 0 & 1 & 1\end{array}$ &,- 3 &,-- & 6,10 & 6,3 & 7,12 & 4,3 & 4,3 & 6,7 \\
\hline $\begin{array}{llllllll}0 & 1 & 1 & 0\end{array}$ &,-- &,-- & 17,16 &,- 10 & 18,16 & 11,9 & 11,8 & 17,13 \\
\hline $\begin{array}{llll}0 & 1 & 0 & 1\end{array}$ &,- 4 &,-- & 12,10 & 15,4 & 10,12 & 5,5 & 6,5 & 9,9 \\
\hline $\begin{array}{llll}1 & 0 & 1 & 0\end{array}$ &,- 4 &,-- & 5,11 & 7,4 & 6,11 & 5,5 & 4,4 & 6,8 \\
\hline $\begin{array}{llll}10 & 0 & 1\end{array}$ &,- 3 &,--- & 5,10 & 6,3 & 7,11 & 4,3 & 4,3 & 6,8 \\
\hline 1100 &,-- &,-- & 15,16 &,- 15 & 17,16 & 12,13 & 12,10 & 17,12 \\
\hline $\begin{array}{llll}1 & 1 & 1 & 1\end{array}$ &,- 5 &,-- & 14,11 &,- 4 & 14,14 & 6,6 & 6,4 & 10,9 \\
\hline $\mathbf{x}$ &,-- &,-- &,-- &,- 14 &,-- & $16,-$ & 14,14 & 17,20 \\
\hline
\end{tabular}

effective here as a final stage ${ }^{1}$. In table II, PA improves upon P-stage performance in $8 / 18$ cases ( 6 reaching global minimum values) while retaining the 9 minima. However, applied to PR, only 6 cases are improved, the other 12 having already reached minimum values. In III, PA improves upon the P-stage in $12 / 18$ cases (6 reaching global minimum values) although only retaining 2 minima, the other 2 marginally worsened. Finally, PRA improves upon PR in $11 / 18$ cases (10 becoming global minima), retaining 6 global minima and providing the strongest performance overall. Thus it can be taken that PR is the more effective pre-conditioner to the final A-stage.

Tables IV and V show the logarithmic convergence of Relative Root Mean Square Error (RRMSE), Relative Maximum Absolute Error (RMAE) and $R^{2}$ values with increasing $N$ for each method across the design domain $\mathbf{x}$. It can be seen that any associated cost benefit relative to the HFSM decreases in proportion to $N$, the HFSM eventually approaching superiority at around 20HF (although note the exponentially worse performance at lower $N$ given the lack of LF trend data). It is likely then that for each case, there exists a critical value of $N$, above which the HFSM will always offer the superior approximation. However, this value is likely dependant on the sampling strategy employed. For example, an adaptive sampling strategy that refines the MF approximation by exploiting areas of predicted improvement and/or maximum discrepancy to improve local accuracy may further increase the attainable prediction quality relative to the overall number of samples. Thus the decline in cost advantage relative to pure HF meta-modelling may be tempered.

\section{Cost}

This section presents a brief cost report based on the recommendations of [47]. Results were obtained over 100 independent runs using a $2.5 \mathrm{GHz}$ Intel(R) Core(TM) i5-7200 CPU with 8GB RAM. Table VI displays model costs and error ratios. Average run-times recorded for FOSTRAD and SU2 were 9.65 s and 3110 s respectively. Geometry generation accounts for an additional $0.83 \mathrm{~s}$ for a $2 \mathrm{D}$ surface mesh and $17.64 \mathrm{~s}$ for a 3D volume mesh. The average run-time of a trained ANN surrogate was found to be 1.9951e-4s. Fig. 3 shows the average time required to perform the MF correction

\footnotetext{
${ }^{1}$ Additive correction quality is inversely proportional to discrepancy variation [46]. A poorly scaled LF response is unlikely to correct well given a limited HF sample budget (e.g. RA offers little improvement over A).
} 
TABLE IV: $\mathbf{x}$ domain prediction accuracy at fixed costs $\left[C_{L}, C_{D}\right]$

\begin{tabular}{|c|c|c|c|c|c|c|c|c|}
\hline & \multicolumn{2}{|c|}{$5 \mathrm{HF}$} & \multicolumn{2}{|c|}{$10 \mathrm{HF}$} & \multicolumn{2}{|c|}{$15 \mathrm{HF}$} & \multicolumn{2}{|c|}{$20 \mathrm{HF}$} \\
\hline & RRMSE & $R M A E$ & RRMSE & $R M A E$ & RRMSE & $R M A E$ & RRMSE & $R M A E$ \\
\hline $\mathrm{P}$ & $0.3681, \mathbf{0 . 2 7 8 4}$ & $1.440,1.229$ & $0.3414,0.2556$ & $1.422,1.151$ & $0.3284,0.2317$ & $1.416,1.107$ & $0.3222,0.2337$ & $1.414,1.102$ \\
\hline $\mathrm{R}$ & $0.6898,0.9099$ & $1.908,3.152$ & $0.6521,0.8573$ & $1.794,3.080$ & $0.6179,0.8035$ & $1.668,2.847$ & $0.5927,0.7735$ & $1.558,2.721$ \\
\hline A & $0.6412,0.9756$ & $1.874,3.172$ & $0.3694,0.7309$ & $1.272,2.360$ & $0.3238,0.5026$ & $1.145,1.844$ & $0.2750,0.3753$ & $1.091,1.465$ \\
\hline PR & $0.3428,0.2806$ & $1.391,1.228$ & $0.3070, \mathbf{0 . 2 4 5 9}$ & $1.347,1.1340$ & $0.2839, \mathbf{0 . 2 1 9 4}$ & $1.312,1.074$ & $0.2740,0.2151$ & $1.293,1.055$ \\
\hline RA & $0.6432,0.8894$ & $1.845,3.115$ & $0.3669,0.7073$ & $1.218,2.553$ & $0.3124,0.5008$ & $1.150,1.944$ & $0.2682,0.3936$ & $1.071,1.590$ \\
\hline PA & $0.3419,0.3126$ & 1.311, 1.221 & $0.2664,0.3052$ & $\mathbf{1 . 0 8 1}, 1.125$ & $0.2242,0.2698$ & $\mathbf{0 . 9 9 2 9}, 1.020$ & $0.2057,0.2410$ & $0.9306, \mathbf{0 . 9 6 4 8}$ \\
\hline PRA & $\mathbf{0 . 3 3 0 2}, 0.2856$ & $1.339, \mathbf{1 . 2 1 6}$ & $\mathbf{0 . 2 3 7 7}, 0.2635$ & $1.120, \mathbf{1 . 0 8 7}$ & $\mathbf{0 . 2 0 7 7}, 0.2347$ & $1.017, \mathbf{0 . 9 7 9 2}$ & $\mathbf{0 . 1 8 8 0}, 0.2301$ & $0.9458,0.9690$ \\
\hline HFSM & $0.6653,0.7166$ & $1.896,2.148$ & $0.2917,0.4247$ & $1.120,1.440$ & $0.2365,0.2808$ & $1.003,1.126$ & $0.2007, \mathbf{0 . 2 0 5 6}$ & $\mathbf{0 . 9 1 9 1}, 0.9730$ \\
\hline
\end{tabular}

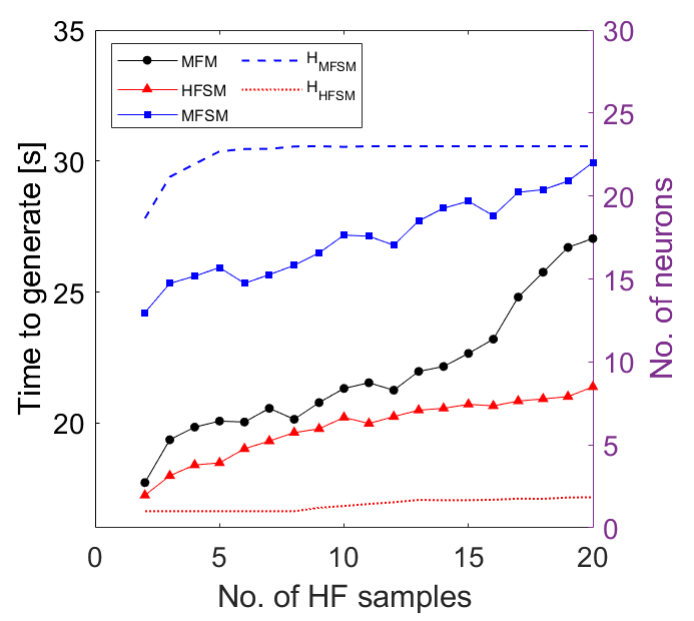

Fig. 3: Surrogate generation resource

and generate the HFSM/MFSM, including the average value of $H$ determined by the double-loop training procedure.

\section{CONCLUSION}

This paper presents a MFSM generation method intended to provide global approximations of an expensive function for use within many-query/outer-loop applications. The method is examined with respect to individual and sequential application of three constituent corrective stages. The method is used to predict the aerodynamic response of a parametrized waveriderbased re-entry vehicle. The 3-stage (PRA) configuration is found to provide an accurate and stable representation of the vehicle lift and drag coefficients in fewer HF samples compared to selected single and 2-stage methods. Furthermore, the method is found to outperform pure HF meta-modelling

TABLE V: $R^{2}[\%]$ at fixed costs $\left[C_{L}, C_{D}\right]$

\begin{tabular}{|c|c|c|c|c|}
\hline Method & $\mathbf{5 H F}$ & $\mathbf{1 0 H F}$ & $\mathbf{1 5 H F}$ & $\mathbf{2 0 H F}$ \\
\hline P & $84.56,92.05$ & $86.90,93.41$ & $87.33,94.10$ & $87.47,94.07$ \\
R & $79.68,74.05$ & $81.60,76.46$ & $82.77,78.14$ & $83.66,79.08$ \\
A & $78.54,66.00$ & $87.16,69.21$ & $89.44,80.82$ & $92.34,87.46$ \\
PR & $87.19, \mathbf{9 2 . 1 5}$ & $89.75, \mathbf{9 3 . 9 9}$ & $90.71, \mathbf{9 4 . 7 9}$ & $91.24,95.00$ \\
RA & $80.53,72.84$ & $90.46,76.01$ & $91.77,84.12$ & $93.79,88.91$ \\
PA & $87.56,90.42$ & $92.20,90.61$ & $94.62,92.66$ & $95.49,94.30$ \\
PRA & $\mathbf{8 8 . 3 9 ,} 92.06$ & $\mathbf{9 4 . 0 0}, 93.26$ & $\mathbf{9 5 . 3 8}, 94.33$ & $\mathbf{9 6 . 2 2}, 94.71$ \\
HFSM & $-5.12,-15.44$ & $89.75,78.76$ & $93.33,90.89$ & $95.62, \mathbf{9 5 . 2 0}$ \\
\hline
\end{tabular}

TABLE VI: Cost/accuracy report

\begin{tabular}{|l|l|}
\hline Property & Value \\
\hline RRMSE LF/HF $\left[C_{L}, C_{D}\right]$ & $1.564,1.328$ \\
RMAE LF/HF $\left[C_{L}, C_{D}\right]$ & $3.581,4.397$ \\
$R^{2} \mathrm{LF} / \mathrm{HF}\left[C_{L}, C_{D}\right]$ & $17.35 \%, 55.94 \%$ \\
Cost LF/HF & 0.0031 \\
Cost SM/LF & $2.0671 \mathrm{e}-05$ \\
Cost SM/HF & $6.4151 \mathrm{e}-08$ \\
\hline
\end{tabular}

for sample sizes lower than a problem-dependant critical value. Unsurprisingly, correction quality is found to be largely influenced by model correlation. Further work will aim to quantify the capabilities of each stage relative to types of geometric discrepancy observable in function responses. Diminishing performance gains with increasing samples suggests the need for an adaptive sampling procedure to preserve cost benefits until the desired local accuracy is achieved. This may be accomplished via iterative refinement within regions of interest/expected improvement following the generation of an acceptable global approximation. The incorporation of the proposed methodology into a MF model management framework and the application to a multi-objective design optimisation problem will be presented in a future work.

\section{ACKNOWLEDGMENT}

Section VI results were obtained using the ARCHIE-WeSt High Performance Computer (www.archie-west.ac.uk).

\section{REFERENCES}

[1] T. W. Simpson, "Design and Analysis of Computer Experiments in Multidisciplinary Design Optimization: A Review of How Far We Have Come - or Not," AIAA, no. September, pp. 1-21, 2008.

[2] C. C. Fischer, R. V. Grandhi, and P. S. Beran, "Bayesian-enhanced lowfidelity correction approach to multifidelity aerospace design," AIAA Journal, vol. 56, no. 8, pp. 3295-3306, 2018.

[3] D. E. Bryson, R. Haimes, and J. F. Dannenhoffer, "Toward the realization of a highly integrated, multidisciplinary, multifidelity design environment," AIAA Scitech 2019 Forum, no. January, pp. 1-15, 2019.

[4] D. S. Lazzara, R. Haimes, and K. Willcox, "Multifidelity geometry and analysis in aircraft conceptual design," 19th AIAA Computational Fluid Dynamics Conference, no. June, pp. 1-22, 2009.

[5] D. Allaire and K. Willcox, "A mathematical and computational framework for multifidelity design and analysis with computer models," International Journal for Uncertainty Quantification, vol. 4, no. 1, pp. $1-20,2014$.

[6] R. R. Schaller, "Moore's law: past, present, and future," IEEE Spectrum, vol. 34, no. 6, pp. 52-55, 57, 1997.

[7] S. Venkataraman and R. T. Haftka, "Structural optimization complexity: What has Moore's law done for us?" Structural and Multidisciplinary Optimization, vol. 28, no. 6, pp. 375-387, 2004. 
[8] M. G. Fernández-Godino, C. Park, N.-H. Kim, and R. T. Haftka, "Review of multi-fidelity models," 2016. [Online]. Available: http://arxiv.org/abs/1609.07196

[9] S. Koziel and L. Leifsson, Simulation-driven design by knowledge-based response correction techniques, 2016.

[10] Q. Zhou, Y. Wang, S. K. Choi, P. Jiang, X. Shao, J. Hu, and L. Shu, "A robust optimization approach based on multi-fidelity metamodel," Structural and Multidisciplinary Optimization, vol. 57, no. 2, pp. 775797, 2018.

[11] B. Peherstorfer, K. Willcox, and M. Gunzburger, "Survey of multifidelity methods in uncertainty propagation, inference, and optimization," SIAM Review, vol. 60, no. 3, pp. 550-591, 2018.

[12] M. Shi, L. Lv, W. Sun, and X. Song, "A multi-fidelity surrogate model based on support vector regression," Structural and Multidisciplinary Optimization, 2020.

[13] S. Choi, J. J. Alonso, and I. M. Kroo, "Multi-fidelity design optimization studies for supersonic jets using surrogate management frame method,' Collection of Technical Papers - AIAA Applied Aerodynamics Conference, vol. 2, pp. 960-977, 2005.

[14] I. M. Jonsson, L. Leifsson, S. Koziel, Y. A. Tesfahunegn, and A. Bekasiewicz, "Shape optimization of trawl-doors using variablefidelity models and space mapping," Procedia Computer Science, vol. 51, no. 1, pp. 905-913, 2015.

[15] R. Vitali, O. Park, R. T. Haftka, B. V. Sankar, and C. A. Rose, "Structural optimization of a hat-stiffened panel using response surfaces," Journal of aircraft, vol. 39, no. 1, pp. 158-166, 2002.

[16] A. I. Forrester, "Black-box calibration for complex-system simulation," Philosophical Transactions of the Royal Society A: Mathematical, Physical and Engineering Sciences, vol. 368, no. 1924, pp. 3567-3579, 2010.

[17] M. K. Zahir and Z. Gao, "Variable-fidelity optimization with design space reduction," Chinese Journal of Aeronautics, vol. 26, no. 4, pp. 841-849, 2013. [Online]. Available: http://dx.doi.org/10.1016/j.cja.2013.06.002

[18] R. Yondo, E. Andrés, and E. Valero, "A review on design of experiments and surrogate models in aircraft real-time and many-query aerodynamic analyses," Progress in Aerospace Sciences, vol. 96, no. November 2017, pp. 23-61, 2018.

[19] S. E. Gano, J. E. Renaud, and B. Sanders, "Hybrid variable fidelity optimization by using a kriging-based scaling function," AIAA Journal, vol. 43, no. 11, pp. 2422-2430, 2005.

[20] T. Goel, R. T. Haftka, W. Shyy, and N. V. Queipo, "Ensemble of surrogates," Structural and Multidisciplinary Optimization, vol. 33, no. 3, pp. 199-216, 2007.

[21] B. Peherstorfer, T. Cui, Y. Marzouk, and K. Willcox, "Multi-fidelity Monte Carlo: importance sampling method using high-fidelity and surrogate model," vol. 7831, pp. 1-23, 2015.

[22] A. J. Keane, "Wing optimization using design of experiment, response surface, and data fusion methods," Journal of Aircraft, vol. 40, no. 4, pp. 741-750, 2003.

[23] N. M. Alexandrov, R. M. Lewis, C. R. Gumbert, L. L. Green, and P. A. Newman, "Approximation and model management in aerodynamic optimization with variable-fidelity models," Journal of Aircraft, vol. 38 no. 6, pp. 1093-1101, 2001.

[24] Z. Han, C. Xu, L. Zhang, Y. Zhang, K. Zhang, and W. Song, "Efficient aerodynamic shape optimization using variable-fidelity surrogate models and multilevel computational grids," Chinese Journal of Aeronautics, vol. 33, no. 1, pp. 31-47, 2020. [Online]. Available: https://doi.org/10.1016/j.cja.2019.05.001

[25] Q. Zhou, J. Wu, T. Xue, and P. Jin, "A two-stage adaptive multifidelity surrogate model-assisted multi-objective genetic algorithm for computationally expensive problems," Engineering with Computers, no. 0123456789, 2019. [Online]. Available: https://doi.org/10.1007/s00366019-00844-8

[26] D. Gorissen, I. Couckuyt, P. Demeester, T. Dhaene, and K. Crombecq "A surrogate modeling and adaptive sampling toolbox for computer based design," Journal of Machine Learning Research, vol. 11, pp. 2051-2055, 2010

[27] A. Cozad and N. V. Sahinidas, "Learning surrogate models for simulation-based optimization," AIChE Journal, vol. 60, pp. 2211-2227, 2014.

[28] T. Lukaczyk, A. D. Wendor, E. Boteroz, T. Macdonaldz, T. Momosez, A. Variyarz, J. M. Veghz, M. Colonnox, T. D. Economon, J. J. Alonsok, T. H. Orra, and C. I. Da Silvayy, "SUAVE: An open-source environment for multi-fidelity conceptual vehicle design," 16th AIAA/ISSMO Multi- disciplinary Analysis and Optimization Conference, no. June, pp. 1-56, 2015.

[29] M. H. Beale, M. T. Hagan, and H. B. Demuth, Neural Network Toolbox тм User's Guide R2017b, 2017.

[30] M. Santos, B. Mattos, and R. Girardi, "Aerodynamic coefficient prediction of airfoils using neural networks," in 46th AIAA aerospace sciences meeting and exhibit, 2008, p. 887.

[31] J. Roshanian, A. A. Bataleblu, and M. Ebrahimi, "A novel metamodel management strategy for robust trajectory design of an expendable launch vehicle," Proceedings of the Institution of Mechanical Engineers Part G: Journal of Aerospace Engineering, vol. 0, no. 0, pp. 1-18, 2019.

[32] E. Minisci and V. Massimiliano, "Robust Design of a Re-entry Unmanned Space Vehicle by Multi-Fidelity Evolution Control," vol. 51, pp. 4-6, 2013.

[33] T. Mengistu and W. Ghaly, "Aerodynamic optimization of turbomachinery blades using evolutionary methods and ANN-based surrogate models," Optimization and Engineering, vol. 9, no. 3, pp. 239-255, 2008.

[34] R. M. Paiva, A. R. Carvalho, C. Crawford, and A. Suleman, "Comparison of surrogate models in a multidisciplinary optimization framework for wing design," AIAA Journal, vol. 48, no. 5, pp. 995-1006, 2010.

[35] M. H. Bakr, J. W. Bandler, K. Madsen, J. E. Rayas-Sanchez, and J. Sondergaard, "Space mapping optimization of microwave circuits exploiting surrogate models," IEEE MTT-S International Microwave Symposium Digest, vol. 3, no. June, pp. 1785-1788, 2000.

[36] S. J. Leary, A. Bhaskar, and A. J. Keane, "A knowledge-based approach to response surface modelling in multifidelity optimization," Journal of Global Optimization, vol. 26, no. 3, pp. 297-319, 2003

[37] G. Heath, "Mathworks Community Profile - Greg Heath." [Online]. Available: https://uk.mathworks.com/matlabcentral/profile/authors/2929937greg-heath

[38] A. N. Kolmogorov, "On the representation of continuous functions of several variables as superpositions of continuous functions of one variable and addition," Doklady Akademii. Nauk USSR, vol. 114, pp. 679-681, 1957.

[39] A. I. Forrester, A. Sóbester, and A. J. Keane, "Multi-fidelity optimization via surrogate modelling," Proceedings of the Royal Society A: Mathematical, Physical and Engineering Sciences, vol. 463, no. 2088, pp. 3251-3269, 2007

[40] R. P. Starkey and M. J. Lewis, "Analytical off-design lift-to-drag-ratio analysis for hypersonic waveriders," Journal of Spacecraft and Rockets, vol. 37, no. 5, pp. 684-691, 2000

[41] C. Geuzaine and J.-F. Remacle, "Gmsh: A 3-D finite element mesh generator with built-in pre-and post-processing facilities," International Journal for Numerical Methods in Engineering, vol. 79, pp. 1309-1331, 2009. [Online]. Available: http://onlinelibrary.wiley.com/doi/10.1002/nme.3279/full

[42] P. M. Mehta, E. Minisci, M. Vasile, A. C. Walker, and M. Brown, "An Open-source Hypersonic Aerodynamic And Aerothermodynamic Modeling Tool," 8th European Symposium on Aerothermodynamics for Space Vehicles, no. 1, p. 9, 2015.

[43] P. M. Mehta, A. Walker, M. Brown, E. Minisci, and M. L. Vasile, "Sensitivity Analysis towards Probabilistic Re-Entry Modeling of Spacecraft and Space Debris," no. August 2016, 2015.

[44] A. Falchi, E. Minisci, M. Vasile, D. Rastelli, and N. Bellini, "DSMCbased Correction Factor for Low-fidelity Hypersonic Aerodynamics of Re-entering Objects and Space Debris," 7th European Conference of Aeronautics and Aerospace Sciences, vol. 10, no. July, 2017.

[45] F. Palacios, M. R. Colonno, A. C. Aranake, A. Campos, S. R. Copeland, T. D. Economon, A. K. Lonkar, T. W. Lukaczyk, T. W. Taylor, and J. J. Alonso, "Stanford University Unstructured (SU2): An open-source integrated computational environment for multi-physics simulation and design," 51st AIAA Aerospace Sciences Meeting including the New Horizons Forum and Aerospace Exposition 2013, no. January, pp. 160, 2013.

[46] C. Park, R. T. Haftka, and N. H. Kim, "Low-fidelity scale factor improves Bayesian multi-fidelity prediction by reducing bumpiness of discrepancy function," Structural and Multidisciplinary Optimization, vol. 58, no. 2, pp. 399-414, 2018

[47] M. Fernández-Godino, C. Park, N. H. Kim, and R. T. Haftka, "Issues in deciding whether to use multifidelity surrogates," AIAA Journal, vol. 57, no. 5, pp. 2039-2054, 2019. 